\title{
Mismatch repair deficiency may affect clinical outcome through immune response activation in metastatic gastric cancer patients receiving first-line chemotherapy
}

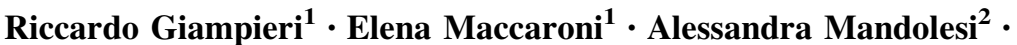 \\ Michela Del Prete $^{1} \cdot$ Kalliopi Andrikou $^{1} \cdot$ Luca Faloppi $^{1} \cdot$ Alessandro Bittoni $^{1}$ • \\ Maristella Bianconi $^{1} \cdot$ Marina Scarpelli $^{2} \cdot$ Raffaella Bracci $^{1} \cdot$ Mario Scartozzi $^{3}$ • \\ Stefano Cascinu ${ }^{1}$
}

Received: 16 October 2015/Accepted: 9 January 2016/Published online: 21 January 2016

(c) The International Gastric Cancer Association and The Japanese Gastric Cancer Association 2016

\begin{abstract}
Introduction The microsatellite-instable gastric cancer subtype, because of its supposed high antigenic potential, is a promising candidate for immunotherapy. We analyzed if the presence of a defective mismatch repair (MMR) system is associated with other markers of immune response and their relationship with outcome in advanced gastric cancer patients.

Methods We analyzed the relationship between clinical outcome and MMR status, the presence of tumor-infiltrating lymphocytes (TIL), lymphocytosis, and neutrophil-tolymphocyte ratio (NLR) in metastatic gastric cancer patients treated with a chemotherapy doublet in the firstline setting. Other stratification factors were sex, age, Eastern Cooperative Oncology Group performance status, adjuvant/neoadjuvant chemotherapy, metastatic sites, and histotype.

Results One hundred three patients were eligible for analysis. Defective MMR was found in 15 patients (14\%), TILs were found in 18 patients (17\%), lymphocytosis was found in 24 patients ( $23 \%$ ), and high NLR was found in 75
\end{abstract}

Electronic supplementary material The online version of this article (doi:10.1007/s10120-016-0594-4) contains supplementary material, which is available to authorized users.

Elena Maccaroni

elena.maccaroni@live.it

1 Department of Medical Oncology, Azienda Ospedaliero Universitaria Ospedali Riuniti, Università Politecnica delle Marche, Ancona, Italy

2 Department of Pathology, Università Politecnica delle Marche, Ancona, Italy

3 Department of Medical Oncology, Università di Cagliari, Cagliari, Italy patients $(72 \%)$. Significant correlations were found between defective MMR and TIL positivity $(p=0.0004)$, between defective MMR and lymphocytosis $(p=0.0062)$, between defective MMR and low NLR $(p=0.000069)$, and between TIL positivity and lymphocytosis $(p=0.000147)$. All factors had a statistically significant impact on overall survival, although on multivariate analysis only defective MMR $(p=0.0001)$ and TIL positivity $(p=0.0192)$ maintained their independent prognostic role. Similar results were observed for progression-free survival, with defective MMR $(p=0.0001)$ and TIL positivity $(p=0.0195)$ maintaining their prognostic role on multivariate analysis.

Conclusions Our analysis confirms the favorable prognosis of metastatic gastric cancer patients with a defective MMR system and suggests that expression of TILs might also be linked to better outcome. Because of the correlation between defective MMR status and measures of immune system activity, this group of patients would be the best candidates for novel immunotherapy-based therapies.

Keywords Gastric cancer - Mismatch repair . Microsatellite instability · Chemotherapy · Prognosis

\section{Introduction}

In Western countries approximately half of gastric tumors are diagnosed at an advanced stage, when a curative surgical resection is no longer possible. As a consequence, in most affected patients palliative chemotherapy is the only treatment option able to improve clinical outcome. Therapeutic approaches have continuously evolved in this setting, particularly with the introduction of targeted agents such as the anti-human epidermal growth factor receptor 2 
monoclonal antibody trastuzumab [1] and the anti-vascular endothelial growth factor receptor 2 monoclonal antibody ramucirumab [2, 3]. Apart from human epidermal growth factor receptor 2 characterization, the issue of treatment selection based on clinical and biological factors is still pending, and we are essentially unable to select the optimal option for each patient.

Recently, researchers from The Cancer Genome Atlas [4] proposed a categorization of gastric cancer into four major subtypes: Epstein-Barr virus related, microsatellite instable (MSI), genomically stable, and chromosomally instable. The MSI category, presenting a typical lack of function of the mismatch repair (MMR) genes (mainly MLH1 and MSH2), is of clinical interest because of the favorable prognostic profile of such tumors compared with their MMR-proficient counterpart.

Several studies have demonstrated that gastric cancer patients lacking MMR gene activity have a better outcome than patients with normal function of the MMR machinery. Similar data have been found in other tumor types, such as colorectal [5-8] and ovarian cancers, thus supporting the observation that MMR-deficient tumors have distinctive biological and clinical characteristics. Intriguingly, recent findings also hypothesized that this favorable clinical behavior might be related to immune response induced by tumor cells in the presence of MMR deficiency. In colorectal cancer cells, preclinical data suggested that the antigenic load able to activate immune response was significantly higher in high microsatellite instability tumors (which is an indirect indication of dysfunctional MMR). Accordingly, only high microsatellite instability colorectal tumors seem to derive a relevant benefit from immunotherapy.

A possible role for immunotherapy has been shown also in the treatment of gastric cancer patients, not assessed according to a specific clinical or molecular profile [9]. On the other hand, the influence of MMR gene status on treatment outcome during chemotherapy [10-13] and the potential connection with indirect signs of immune reaction are still lacking in the published literature.

The aim of this study was to assess the correlation between the loss of MMR activity, signs of immune response activation [presence of tumor-infiltrating lymphocytes (TILs), systemic lymphocytosis, and neutrophilto-lymphocyte ratio (NLR)] and clinical outcome in metastatic gastric cancer patients receiving first-line chemotherapy.

Secondary objective was to delineate a clinical and molecular profile of gastric tumors in which an underlying mechanism of immune-mediated activity may make these patients optimal candidates for immune therapy.

\section{Patients and methods}

\section{Patient selection}

Histologically proven, metastatic gastric cancer patients receiving first-line platinum-based chemotherapy (either cisplatin or oxaliplatin combined with 5-fluorouracil) at our institution between January 2007 and December 2013 and whose tumor sample was available for biological analysis were eligible for our study.

For patients who received adjuvant/neoadjuvant treatment after radical surgical resection, an interval of at least 12 months from the end of treatment was considered necessary for their inclusion in the present analysis. Follow-up during chemotherapy was performed according to our institutional guidelines and consisted of physical examination, full blood count and renal and liver function tests before each cycle. Tumor response was evaluated by a CT scan of the chest and abdomen every 8 weeks assessed by clinicians according to the Response Evaluation Criteria in Solid Tumors (RECIST, version 1.1). The site and date of progression and the date of death were recorded. For all patients, we also collected neutrophil, lymphocyte, and platelet counts within 1 month before the start of treatment. All blood examinations were performed according to International Federation of Clinical Chemistry and Laboratory Medicine method. The assay was conducted in institution laboratories certified for quality control according to the present rules in Europe.

This analysis was approved by the local ethics committee.

\section{Immunohistochemical analysis of mutL homolog 1 and mutS homolog 2}

Immunohistochemical investigation of mutL homolog 1 (MLH1) and mutS homolog 2 (MSH2) expression was performed on paraffin-embedded tissue sections. Five-micrometer-thick sections containing tumor tissue and normal gastric mucosa (internal control) were removed from the paraffin and rehydrated with xylene and alcohol. We blocked endogenous peroxidase by dipping the sections in $3 \%$ aqueous $\mathrm{H}_{2} \mathrm{O}_{2}$ for $10 \mathrm{~min}$, and antigen retrieval was performed with a 10 -min microwave treatment in $10 \mathrm{mmol} /$ $\mathrm{L}$ citrate buffer, $\mathrm{pH}$ 6.00. After antigen retrieval, sections were incubated overnight at $4{ }^{\circ} \mathrm{C}$ with a mouse monoclonal antibody to MLH1 (clone G168-728, 1:50 dilution; PharMingen, San Diego, CA, USA) or to MSH2 (clone FE11, 1:100 dilution; Oncogene Research Products, Cambridge, MA, USA) and lightly counterstained with hematoxylin. Immunostaining was performed by the avidinbiotin peroxidase complex technique; diaminobenzidine 
was used as a chromogen. The normal staining pattern for both MLH1 and MSH2 was assessed, and tumors were considered negative for MSH2 or MLH1 expression when there was a complete absence of nuclear staining of tumor cells in the presence of an internal positive control represented by normal epithelial cells, stromal cells, or lymphocytes.

Tumors with lack of either MLH1 or MSH2 expression were considered MMR defective, whereas tumors that maintained expression of MLH1 and MSH2 were considered MMR proficient.

\section{Tumor-infiltrating lymphocytes}

All tumor samples were routinely evaluated for TILs by our pathologists and were classified as TIL positive or TIL negative. Tumors were considered TIL positive if there was documented evidence of lymphocytic response, assessed by the presence of $\mathrm{CD}^{+}$cells in the histologic specimen. In particular, evaluation and scoring of TIL expression was assessed by the method described by Denkert et al. [14]. The presence of more than 50-60\% stromal surface area covered by TILs was considered as positive. No evaluation of immune cell type was performed.

\section{Statistical analysis}

Statistical analysis was performed with the MedCalc version 14.10.2 (MedCalc Software, Ostend, Belgium; https:// www.medcalc.org).

The association between categorical variables was estimated by Fisher's exact test for binomial categorical variables and by the chi-square test for all remaining instances. Survival distribution was estimated by the Kaplan-Meier method. For statistical analysis, overall survival (OS) and progression-free survival (PFS) were defined, respectively, as the interval between the start of first-line chemotherapy to death or the last follow-up visit and as the interval between the start of first-line chemotherapy to clinical progression or death or the last follow-up visit if there was no progression. Significant differences in the probability of relapse between the strata were evaluated by the log-rank test. Hazard ratios (HRs) for median PFS and OS between groups were estimated from Cox regression models.

Cutoff values for lymphocytosis and NLR were determined by receiver operating characteristic curve (ROC) analysis in the patients included in the analysis.

The multivariate analysis also included adjustments for other variables, such as sex (male vs female), age (younger than 65 years vs 65 years or older), Eastern Cooperative Oncology Group (ECOG) performance status (0-1 vs 2 or greater), previous adjuvant/neoadjuvant chemotherapy (yes vs no), and number of metastatic sites ( 1 vs 2 or greater). A significance level of 0.05 was chosen to assess the statistical significance. The Holm-Sidak correction was used to adjust multiple-comparison values.

\section{Results}

One hundred three patients were eligible for our analysis. For the entire cohort, the median OS was 8.8 months [95\% confidence interval (CI) 7.9-9.0 months, range 2.9-18.6 months], whereas the median PFS was 5.8 months $(95 \%$ CI 4.9-6.0 months, range 0.3-15.6 months). Twenty-seven patients $(26 \%)$ achieved partial remission, 58 patients $(56 \%)$ achieved disease stabilization, and in the remaining 18 patients $(17 \%)$ there was progression during treatment.

Forty-six patients $(45 \%)$ had a synchronous metastatic disease at the start of first-line treatment, whereas 57 patients $(55 \%)$ already had surgery for a primary gastric cancer and had started first-line treatment after disease relapse.

The main characteristics of the patients are summarized in Table 1.

Of 103 patients, analysis of MMR expression and TIL expression was performed on histological tissue from previous gastric cancer biopsies in 30 patients (29\%), whereas in the remaining 73 patients $(71 \%)$, the analysis was performed on histological tissue from a previous surgical resection of the primary tumor.

Fifteen patients (14\%) showed loss of MMR expression (lack of expression of MLH1/MSH2 in the tumor). In this group of patients compared with patients with MMR-proficient tumors, significantly improved OS was seen (median OS 14.2 months vs 8.0 months, HR for death 0.24 , $95 \%$ CI $0.16-0.35, p<0.0001$ ) (Fig. 1). An improved PFS was also observed (median PFS 11.2 months vs 5.0 months, HR for progression $0.25,95 \%$ CI $0.17-0.33$, $p<0.0001$ ) (not shown). The response rate was also better in MMR-defective patients, with $66 \%$ (10 patients) achieving a partial response, compared with $19 \%$ (17 patients) for patients with MMR-proficient tumors $(p=0.0004)$.

Eighteen patients $(17 \%)$ showed TILs on pathology examination (examples of expression can be seen in Fig. S1). The median OS was significantly better in TILpositive patients than in TIL-negative patients (median OS 12.5 months vs 7.9 months, HR for death $0.39,95 \% \mathrm{CI}$ 0.26-0.58, $p<0.0001$ ) (Fig. 2). An improved PFS was also evident (median PFS 8.0 months vs 4.9 months, HR for progression $0.40,95 \%$ CI $0.26-0.60, p=0.0001$ ) (not shown). Eighty-three percent of patients (15 patients) exhibiting pathologic TILs achieved partial remission, whereas only $14 \%$ of patients (12 patients) negative for TILs achieved partial remission $(p<0.0001)$. 
Table 1 Patient' characteristics stratified by defective mismatch repair $(D-M M R) /$ proficient mismatch repair $(P-M M R)$ status and by tumorinfiltrating lymphocyte (TIL) status

\begin{tabular}{|c|c|c|c|c|c|c|c|}
\hline & Entire group & D-MMR & P-MMR & $p$ & TIL+ & TIL- & $p$ \\
\hline \multicolumn{8}{|l|}{ Sex } \\
\hline Male & $71(68 \%)$ & $12(80 \%)$ & $59(67 \%)$ & \multirow[t]{2}{*}{0.38} & $11(61 \%)$ & $60(70 \%)$ & \multirow[t]{2}{*}{0.42} \\
\hline Female & $32(32 \%)$ & $3(20 \%)$ & $29(33 \%)$ & & $7(39 \%)$ & $25(30 \%)$ & \\
\hline \multicolumn{8}{|l|}{ Age (years) } \\
\hline$<65$ & $60(58 \%)$ & $8(53 \%)$ & $52(59 \%)$ & \multirow[t]{2}{*}{0.78} & $11(61 \%)$ & $49(57 \%)$ & \multirow[t]{2}{*}{$1(0.993)$} \\
\hline$\geq 65$ & $43(42 \%)$ & $7(47 \%)$ & $36(41 \%)$ & & $7(39 \%)$ & $36(43 \%)$ & \\
\hline \multicolumn{8}{|l|}{ ECOG PS } \\
\hline 0 & $88(85 \%)$ & $13(87 \%)$ & $75(85 \%)$ & \multirow[t]{2}{*}{1} & $16(89 \%)$ & $72(85 \%)$ & \multirow[t]{2}{*}{1} \\
\hline$\geq 1$ & $15(15 \%)$ & $2(13 \%)$ & $13(15 \%)$ & & $2(11 \%)$ & $13(15 \%)$ & \\
\hline \multicolumn{8}{|c|}{ Previous adjuvant/neoadjuvant treatment } \\
\hline Yes & $29(28 \%)$ & $5(33 \%)$ & $24(27 \%)$ & \multirow[t]{2}{*}{0.75} & $7(38 \%)$ & $22(26 \%)$ & \multirow[t]{2}{*}{0.27} \\
\hline No & $74(72 \%)$ & $10(67 \%)$ & $64(73 \%)$ & & $11(62 \%)$ & $63(74 \%)$ & \\
\hline \multicolumn{8}{|l|}{ Number of metastatic sites } \\
\hline 1 & $32(31 \%)$ & $7(46 \%)$ & $25(28 \%)$ & \multirow[t]{2}{*}{0.22} & $8(44 \%)$ & $24(28 \%)$ & \multirow[t]{2}{*}{0.26} \\
\hline$\geq 2$ & $71(69 \%)$ & $8(54 \%)$ & $63(72 \%)$ & & $10(56 \%)$ & $61(72 \%)$ & \\
\hline \multicolumn{8}{|l|}{ Histotype } \\
\hline Intestinal & $66(64 \%)$ & $11(73 \%)$ & $55(62 \%)$ & \multirow[t]{2}{*}{0.56} & $15(83 \%)$ & $51(60 \%)$ & \multirow[t]{2}{*}{0.10} \\
\hline Diffuse & $37(36 \%)$ & $4(27 \%)$ & $33(38 \%)$ & & $3(17 \%)$ & $34(40 \%)$ & \\
\hline \multicolumn{8}{|c|}{ Previous surgery with radical intent } \\
\hline Yes & $57(55 \%)$ & $6(40 \%)$ & $51(58 \%)$ & \multirow[t]{2}{*}{0.26} & $7(47 \%)$ & $50(57 \%)$ & \multirow[t]{2}{*}{0.57} \\
\hline No & $46(45 \%)$ & $9(60 \%)$ & $37(42 \%)$ & & $8(53 \%)$ & $38(43 \%)$ & \\
\hline \multicolumn{8}{|l|}{ Metastatic sites $^{\mathrm{a}}$} \\
\hline Liver & $49(47 \%)$ & $7(47 \%)$ & $42(48 \%)$ & \multirow[t]{5}{*}{$0.42 *$} & $10(56 \%)$ & $39(46 \%)$ & \multirow[t]{5}{*}{$0.27 *$} \\
\hline Peritoneum & $31(30 \%)$ & $6(40 \%)$ & $25(28 \%)$ & & $5(28 \%)$ & $26(31 \%)$ & \\
\hline Lung & $16(16 \%)$ & $4(27 \%)$ & $12(14 \%)$ & & $6(33 \%)$ & $10(12 \%)$ & \\
\hline Abdominal lymph nodes & $22(21 \%)$ & $1(7 \%)$ & $21(24 \%)$ & & $2(11 \%)$ & $20(23 \%)$ & \\
\hline Other (ovary, bones, etc.) & $9(9 \%)$ & $2(13 \%)$ & $7(8 \%)$ & & $2(11 \%)$ & $7(\%)$ & \\
\hline
\end{tabular}

EGOG PS Eastern Cooperative Oncology Group performance status

* $p$ was calculated for the entire distribution of patients with metastatic disease

a The total may be greater than $100 \%$ because of patients having more than one site of metastatic involvement

When we compared the results for TIL expression in tumor biopsy samples or resected gastric cancer specimens, we observed some differences in terms of the impact on OS: in particular, in resected gastric cancer samples we observed TIL-positive status in 14 of 73 patients (19\%), whereas in biopsy samples we observed TIL-positive status in four of 30 patients $(13 \%)$. OS for patients in the resected gastric cancer group was 12.5 months versus 7.9 months for TIL-positive versus TIL-negative status (HR 0.37, $95 \%$ CI $0.23-0.59, p=0.0002$ ) (Fig. S2 ). OS for patients in the biopsy group was 9.9 months versus 7.9 months for TIL-positive versus TIL-negative status (HR 0.50, $95 \%$ CI 0.21-1.17, $p=0.1662$ ) (Fig. S3).

On the basis of receiver operating characteristic analysis results, the best cutoff value for lymphocytosis was set at
1.8 the lower limit normal. Twenty-four patients $(23 \%)$ showed lymphocytosis. The median OS was improved in patients with lymphocytosis compared with the remaining patients (median OS 10.8 months vs 7.9 months, HR for death $0.38,95 \%$ CI $0.26-0.57, p<0.0001$ ) (Fig. 3). The median PFS resulted improved as well in patients with lymphocytosis (median PFS 7.8 months vs 4.9 months, HR for progression 0.38, $95 \%$ CI $0.26-0.57, p<0.0001$ ) (not shown). Seventy-nine percent of patients (19 patients) with lymphocytosis achieved partial remission, whereas only $10 \%$ of patients (8 patients) without lymphocytosis showed a response to treatment $(p<0.0001)$.

On the basis of receiver operating characteristic analysis results, the best cutoff value for NLR was set at 0.4. An NLR higher than 0.4 was found in 75 patients $(72 \%)$. The 


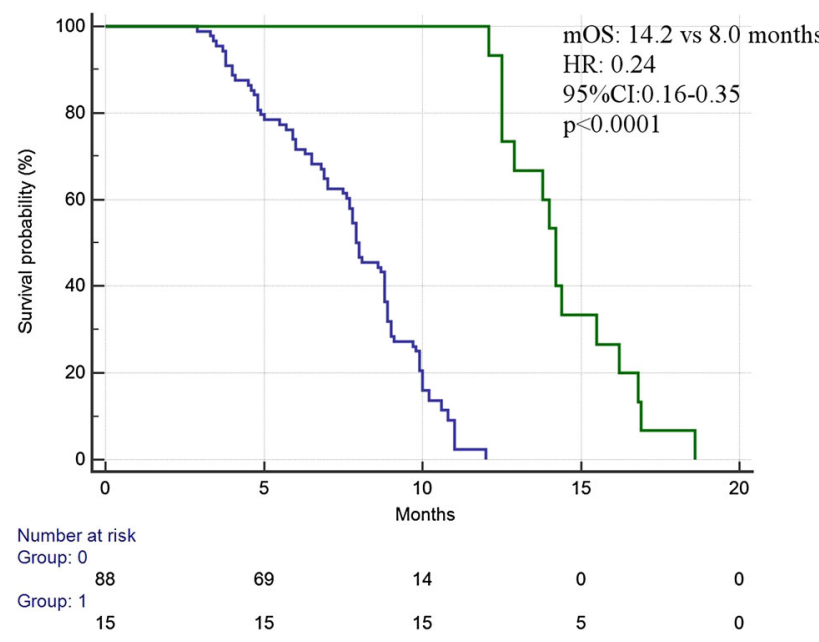

Fig. 1 Overall survival for patients stratified accordingly to mismatch repair (MMR) status. CI confidence interval, $H R$ hazard ratio, mOS median overall survival

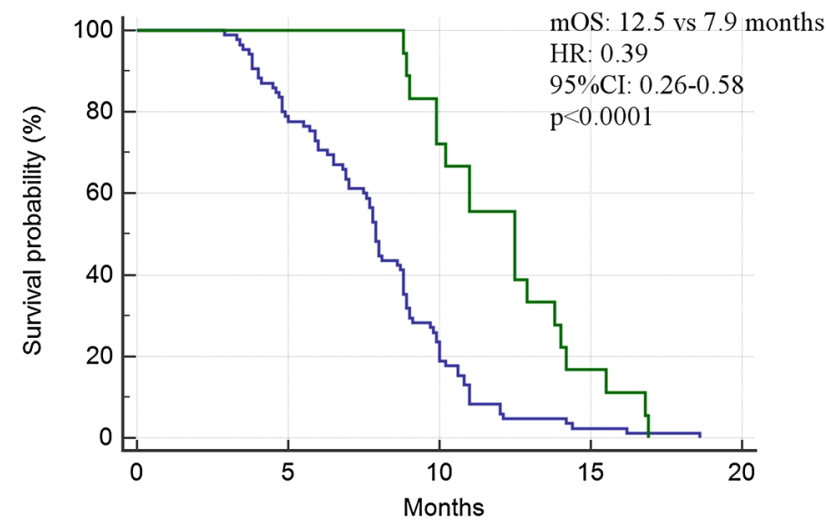

Number at risk

$\begin{array}{ccccc}\text { Group: }{ }_{\text {Group: }}{ }^{1}{ }^{15} & 66 & 16 & 2 & 0 \\ 18 & 18 & 13 & 3 & 0\end{array}$

Fig. 2 Overall survival for patients stratified accordingly to tumorinfiltrating lymphocyte $(T I L)$ expression. $C I$ confidence interval, $H R$ hazard ratio, $m O S$ median overall survival

median OS was significantly worse in this group of patients (median OS 7.8 months vs 10.8 months, HR for death 2.61, $95 \%$ CI 1.77-3.84, $p<0.0001$ ) (Fig. 4). The median PFS was also significantly worse in the high-NLR group versus the low-NLR group (4.8 months vs 7.6 months, HR for progression 2.51, $95 \%$ CI $1.71-3.70, p<0.0001$ ) (not shown). Sixty-seven percent of patients (19 patients) in the low-NLR group achieved a partial response, compared with $11 \%$ of patients (8 patients) in the high-NLR group $(p<0.0001)$.

A significant relationship was found between MMR status and TIL expression. Sixty-six percent of patients (10 patients) with MMR-defective tumors showed concomitant

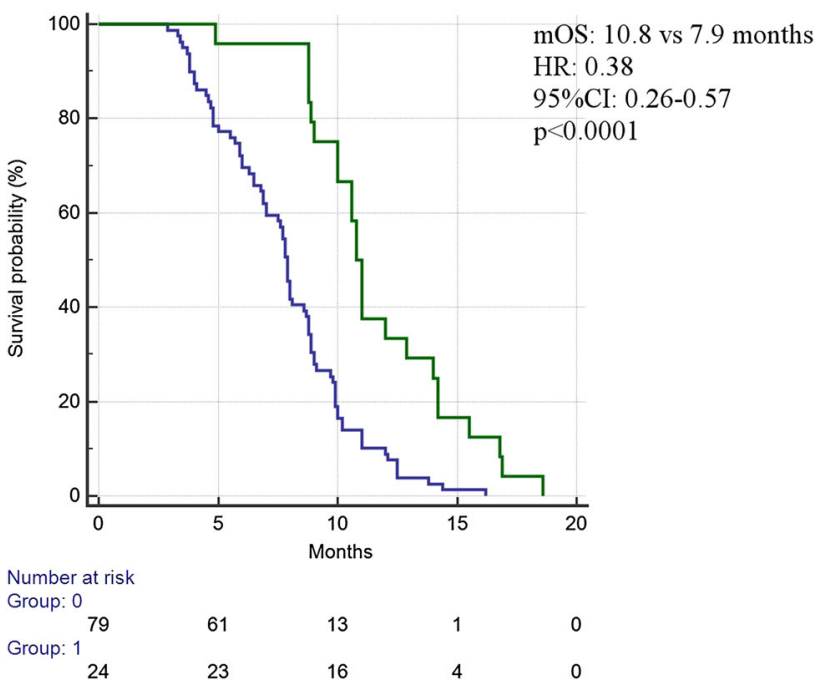

Fig. 3 Overall survival for patients stratified accordingly to lymphocytosis. $C I$ confidence interval, $H R$ hazard ratio, $m O S$ median overall survival

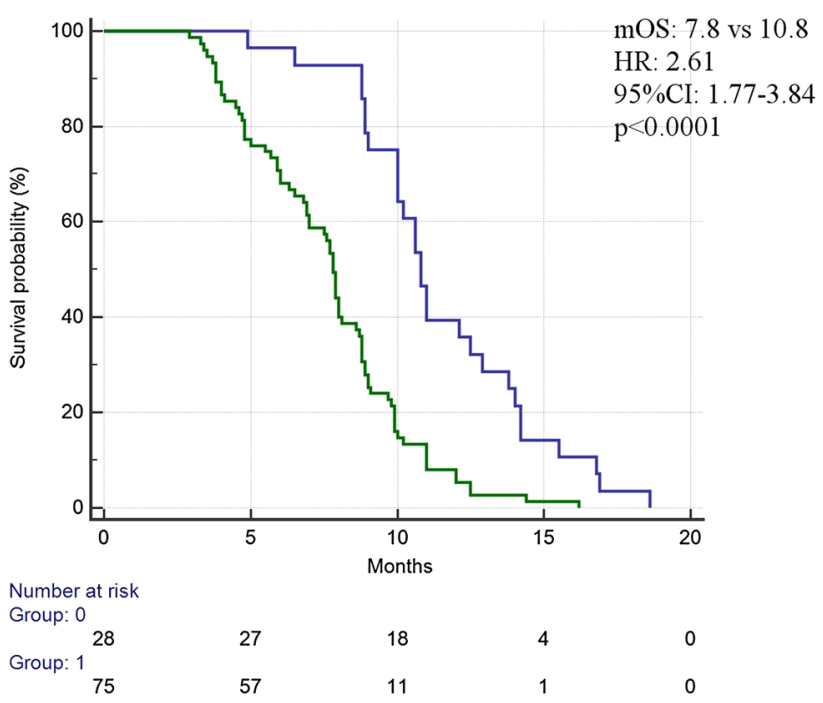

Fig. 4 Overall survival for patients stratified accordingly to neutrophil-to-lymphocyte ratio. $C I$ confidence interval, $H R$ hazard ratio, $m O S$ median overall survival

TILs, compared with $9 \%$ of patients (8 patients) with MMR-proficient tumors $(p=0.0004)$. A significant relationship was also found between MMR status and systemic lymphocytosis. Fifty-three percent of patients (8 patients) with defective MMR also had systemic lymphocytosis, compared with $18 \%$ of patients (6 patients) who had MMR-proficient tumors $(p=0.0062)$. In patients showing TIL expression, systemic lymphocytosis was more frequently associated with it. Sixty-one percent of patients (11 patients) with TILs had systemic lymphocytosis, compared with $15 \%$ of patients (13 patients) without TILs $(p=0.000147)$. An association between MMR status and 


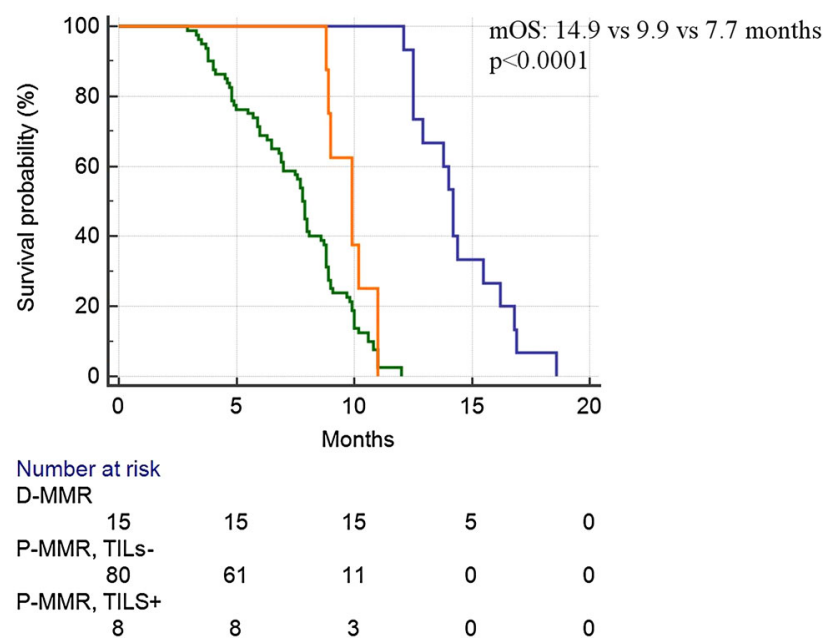

Fig. 5 Overall survival analysis among the three subgroups identified by multivariate analysis. $D-M M R$ defective mismatch repair, $m O S$ median overall survival, $P-M M R$ proficient mismatch repair, TIL tumor-infiltrating lymphocyte

NLR was also found: in particular, only $27 \%$ of patients (4 patients) in the MMR-defective group had a high NLR, compared with $80 \%$ of patients (71 patients) in the MMRproficient group $(p=0.000069)$.

On multivariate analysis, MMR status and TIL expression maintained an independent role for OS and PFS, whereas the other factors (lymphocytosis, NLR, previous adjuvant chemotherapy, ECOG performance status, number of metastatic sites, histotype) were excluded by the model. In particular, MMR-defective status maintained its prognostic role and was shown to be the strongest predictor of better outcome $(\exp B=0.41,95 \%$ CI $0.2528-0.6772$, $p=0.0005)$. TIL-positive status also maintained an independent role as a predictor of better outcome $(\exp B=0.49$, $95 \%$ CI 0.2863-0.8688, $p=0.0145$ ).

On this basis we identified three groups of patients: patients with MMR-defective tumors, patients with MMRproficient tumors and evidence of TILs, and patients with MMR-proficient tumors without evidence of TILs. The median PFS for the three subgroups was 11 months versus 6.9 months versus 4.9 months, respectively, $p<0.0001$ (not shown). Median OS analysis also showed significant differences according to the three subgroups: 14.2 months versus 9.9 months versus 7.7 months, $p<0.0001$ (Fig. 5).

\section{Discussion}

Although treatment options for metastatic gastric cancer have improved, survival is still disappointing. Apart from the need for more effective therapies, a potential explanation for these unsatisfactory results lies in the substantial lack of reliable predictive and prognostic factors, thus limiting the possibility to select the appropriate treatment for appropriate patients. A growing body of evidence is now suggesting that gastric cancer is a highly heterogeneous disease with different biological and clinical features [5]. This ultimately leads to a possibly distinctive prognostic stratification and sensitivity/resistance to therapeutic options: specifically, patients in the Epstein-Barr virus related subgroup appear to be the best candidates to receive treatment options based on immunotherapy and inhibition of the phosphatidylinositol 3-kinase-Akt-mammalian target of rapamycin pathway because of their relatively high frequency of mutations of the PIK3CA gene. On the other hand, although the genomically stable subgroup appears quite homogeneous in its lack of selective mutations (apart from ARIDIA), it would seem to be potentially the worst candidate for a trial based on a targeted agent.

Our analysis confirmed that MMR-defective tumors have an improved clinical outcome compared with their MMR-proficient counterparts. Indeed, we defined MMRdefective tumors on the basis of the lack of expression of MLH1 or MSH2 in the tumor, thus without information regarding the expression (or lack thereof) of MSH6 or $P M S 2$. Although it is well known that MSH6 and PMS2 expression is another crucial factor in the definition of MMR activity in other tumor types (i.e., colon cancer), in gastric cancer it seems that the role of these two genes is less relevant, with $M L H 1$-defective status being the alteration most commonly found in MMR-defective tumors.

Similarly, Shigeyasu et al. [15] suggested that metastatic gastric cancer patients showing $M L H 1$ inactivation via promoter methylation had significantly improved survival. Unsurprisingly $\mathrm{MLH1}$ promoter methylation was also associated with the MSI phenotype as commonly found in all tumor types exhibiting a malfunction of the MMR machinery. A further analysis [16] showed that MSI status and promoter methylation might be associated with improved survival in resected gastric cancer patients, even if the prognostic impact of MMR status was lost on multivariate analysis favoring tumor stage, which is likely to be more prognostically relevant than microsatellite instability.

Our analysis confirmed the prognostic role of MMR status and also indicated a potential role of MMR in predicting the response to first-line platinum-based chemotherapy as suggested by the improvement in response rate and progression-free survival for MMR-defective tumors.

These findings are partly in contrast to previous observations in patients who underwent radical resection receiving adjuvant fluoropyrimidines [17]. However, both the different setting and the type of chemotherapy might explain the conflicting results. 
We also showed that the presence of TILs is an important, independent prognostic factor. This was particularly evident in MMR-deficient tumors, in which the presence of TILs was linked to MMR status and identified a subgroup of patients with a distinctive favorable outcome in terms of PFS and OS.

The clinical implication of TILs in gastric tumors has been long debated since early observations in pathology specimens. Despite the marked heterogeneity of the published literature analyzing TILs in various tumor types [18, 19], there is now a general consensus that TILs are a favorable feature, particularly in locally advanced, radically resected tumors. Nonetheless, data in metastatic patients were lacking-that is, data correlating TILs with response and PFS in this setting. Intriguingly, in our experience TILs are a mixed factor with both a prognostic and a predictive role.

TIL-positive status was more strongly associated with an improved prognosis when the evaluation of TIL scoring was performed on resected gastric cancer samples rather than on tumor biopsy samples: the reason for this difference might be related to the fact that, in tumor biopsy samples, the deeper portion of the tumor, mainly comprising stromal cells (and where we should evaluate TIL expression) could be lacking, thus lessening the reliability of the assessment on simple tumor biopsy samples. Indeed, that might explain why some conflicting results, in terms of the prognostic role of TIL expression, could be seen by comparison of analyses conducted in patients who underwent surgical resection and who are receiving adjuvant treatment compared with those who have metastatic spread since disease onset. This latter group of patients might be "underevaluated" for TIL expression because of the lack of proper tumor tissue, with no resection of their primary tumor usually having been performed.

The option to further classify the cellular components of TILs by means of immunohistochemical markers such as forkhead box P3, CD8, and CD4, to name few, was not possible in our study. However, we believe that our finding of a correlation between MMR status and the presence of TILs is in keeping with our knowledge of the molecular basis underlying MMR-defective tumors and their putative antigenic potential. In fact, we demonstrated that MMRdefective tumors are more frequently associated with TILs and that these peculiar features have a significant impact not only on prognosis but also on RR and PFS. On this basis we can speculate that MMR-defective gastric tumors with TILs might be an interesting area of investigation for the immunotherapy options that are emerging in this setting. Although our study was limited by the small number of patients, it is interesting that all patients who had MMRdefective gastric cancer and TIL expression also had a low
NLR (ten patients), thus hypothetically suggesting an easily obtainable surrogate for a histologic biomarker.

One potential limitation of our work is the relatively small sample size, coupled with the fact that the group of patients who were included in the analysis comprised a heterogeneous population with different types of metastatic gastric cancer (diffuse, intestinal, and mixed subtypes) and who may already have had or may not have had curative surgery before the onset of metastatic disease. This may be at least in part because of this being a monocentric analysis: because of this, some potential bias regarding different treatment regimens used in the metastatic phase, different expertise of the primary treating surgeon, and an overall difference in terms of "behavior" of the oncologist who is primary responsible for patient care, and that might be seen in multicentric analyses, are also lessened.

Our findings confirmed that MMR-defective gastric tumors are a distinctive biological and clinical category and that TILs might be a relevant pathological feature also in the metastatic setting. These two factors appeared to be associated with each other, but were still independently able to influence clinical outcome. On the other hand, the evident correlation between MMR status and the presence of TILs led us to hypothesize that MMR-defective gastric tumors might have an intrinsic antigenic activity potentially relevant for immunotherapy.

In our opinion MMR status should be a stratification factor in future clinical trials investigating treatment options in metastatic gastric cancer patients. This would allow clinicians to individuate specific therapeutic opportunities in specific patient subsets, hopefully improving global results. In particular, the unexplored area of immunotherapy in MMR-defective, TIL-positive gastric tumors might allow better investigation of this novel, although promising, strategy.

\section{Compliance with ethical standards}

Conflict of interest The authors declare that they have no conflict of interest.

Human rights statement and informed consent All procedures followed were in accordance with the ethical standards of the responsible committee on human experimentation (institutional and national) and with the Helsinki Declaration of 1964 and later versions. Informed consent or substitute for it was obtained from all patients for their being included in the study.

\section{References}

1. Bang YJ, Van Cutsem E, Feyereislova A, Chung HC, Shen L, Sawaki A, Lordick F, Ohtsu A, Omuro Y, Satoh T, Aprile G, Kulikov E, Hill J, Lehle M, Rüschoff J, Kang YK. Trastuzumab in combination with chemotherapy versus chemotherapy alone 
for treatment of HER2-positive advanced gastric or gastro-oesophageal junction cancer (ToGA): a phase 3, open-label, randomised controlled trial. Lancet. 2010;376(9742):687-97. doi:10. 1016/S0140-6736(10)61121-X. Erratum in: Lancet. 2010;376(9749):1302.

2. Wilke H, Muro K, Van Cutsem E, Oh SC, Bodoky G, Shimada Y, Hironaka S, Sugimoto N, Lipatov O, Kim TY, Cunningham D, Rougier P, Komatsu Y, Ajani J, Emig M, Carlesi R, Ferry D, Chandrawansa K, Schwartz JD, Ohtsu A. Ramucirumab plus paclitaxel versus placebo plus paclitaxel in patients with previously treated advanced gastric or gastro-oesophageal junction adenocarcinoma (RAINBOW): a double-blind, randomised phase 3 trial. Lancet Oncol. 2014;15(11):1224-35. doi:10.1016/S14702045(14)70420-6.

3. Fuchs CS, Tomasek J, Yong CJ, Dumitru F, Passalacqua R, Goswami C, Safran H, dos Santos LV, Aprile G, Ferry DR, Melichar B, Tehfe M, Topuzov E, Zalcberg JR, Chau I, Campbell W, Sivanandan C, Pikiel J, Koshiji M, Hsu Y, Liepa AM, Gao L, Schwartz JD, Tabernero J. Ramucirumab monotherapy for previously treated advanced gastric or gastro-oesophageal junction adenocarcinoma (REGARD): an international, randomised, multicentre, placebo-controlled, phase 3 trial. Lancet. 2014;383(9911):31-9. doi:10.1016/S0140-6736(13)61719-5.

4. Cancer Genome Atlas Research Network. Comprehensive molecular characterization of gastric adenocarcinoma. Nature. 2014;513(7517):202-9. doi:10.1038/nature13480.

5. Gryfe R, Kim H, Hsieh ET, Aronson MD, Holowaty EJ, Bull SB, Redston M, Gallinger S. Tumor microsatellite instability and clinical outcome in young patients with colorectal cancer. N Engl J Med. 2000;342(2):69-77.

6. Popat S, Hubner R, Houlston RS. Systematic review of microsatellite instability and colorectal cancer prognosis. J Clin Oncol. 2005;23(3):609-18.

7. Sinicrope FA, Foster NR, Thibodeau SN, Marsoni S, Monges G, Labianca R, Kim GP, Yothers G, Allegra C, Moore MJ, Gallinger $\mathrm{S}$, Sargent DJ. DNA mismatch repair status and colon cancer recurrence and survival in clinical trials of 5-fluorouracil-based adjuvant therapy. J Natl Cancer Inst. 2011;103(11):863-75.

8. Maccaroni E, Bracci R, Giampieri R, Bianchi F, Belvederesi L, Brugiati C, Pagliaretta S, Del Prete M, Scartozzi M, Cascinu S. Prognostic impact of mismatch repair genes germline defects in colorectal cancer patients: are all mutations equal? Oncotarget. 2015. doi:10.18632/oncotarget.5395.

9. Muro K, Bang YJ, Shankaran V, Geva R, Catenacci DVT, Gupta $\mathrm{S}$, Eder JP, et al. Relationship between PD-L1 expression and clinical outcomes in patients (Pts) with advanced gastric cancer treated with the anti-PD-1 monoclonal antibody pembrolizumab (Pembro; MK-3475) in KEYNOTE-012. J Clin Oncol. 2015;33(3 Suppl):3.
10. Ribic CM, Sargent DJ, Moore MJ, Thibodeau SN, French AJ, Goldberg RM, Hamilton SR, Laurent-Puig P, Gryfe R, Shepherd LE, Tu D, Redston M, Gallinger S. Tumor microsatellite-instability status as a predictor of benefit from fluorouracil-based adjuvant chemotherapy for colon cancer. $\mathrm{N}$ Engl $\mathrm{J}$ Med. 2003;349(3):247-57.

11. Des Guetz G, Schischmanoff O, Nicolas P, Perret GY, Morere JF, Uzzan B. Does microsatellite instability predict the efficacy of adjuvant chemotherapy in colorectal cancer? A systematic review with meta-analysis. Eur J Cancer. 2009;45(10):1890-6.

12. Sargent DJ, Marsoni S, Monges G, Thibodeau SN, Labianca R, Hamilton SR, French AJ, Kabat B, Foster NR, Torri V, Ribic C, Grothey A, Moore M, Zaniboni A, Seitz JF, Sinicrope F, Gallinger S. Defective mismatch repair as a predictive marker for lack of efficacy of fluorouracil-based adjuvant therapy in colon cancer. J Clin Oncol. 2010;28(20):3219-26.

13. Sinicrope FA, Foster NR, Thibodeau SN, Marsoni S, Monges G, Labianca R, Kim GP, Yothers G, Allegra C, Moore MJ, Gallinger $\mathrm{S}$, Sargent DJ. DNA mismatch repair status and colon cancer recurrence and survival in clinical trials of 5-fluorouracil-based adjuvant therapy. J Natl Cancer Inst. 2011;103(11):863-75.

14. Denkert C, Loibl S, Noske A, et al. Tumor-associated lymphocytes as an independent predictor of response to neoadjuvant chemotherapy in breast cancer. J Clin Oncol. 2010;28:105-13.

15. Shigeyasu K, Nagasaka T, Mori Y, Yokomichi N, Kawai T, Fuji T, Kimura K, Umeda Y, Kagawa S, Goel A, Fujiwara T. Clinical significance of MLH1 methylation and CpG Island methylator phenotype as prognostic markers in patients with gastric cancer. PLoS One. 2015;10(6):e0130409. doi:10.1371/journal.pone. 0130409.

16. An C, Choi IS, Yao JC, Worah S, Xie K, Mansfield PF, Ajani JA, Rashid A, Hamilton SR, Wu TT. Prognostic significance of CpG island methylator phenotype and microsatellite instability in gastric carcinoma. Clin Cancer Res. 2005;11(2 Pt 1):656-63.

17. Kim SY, Choi YY, An JY, Shin HB, Jo A, Choi H, Seo SH, Bang HJ, Cheong JH, Hyung WJ, Noh SH. The benefit of microsatellite instability is attenuated by chemotherapy in stage II and stage III gastric cancer: results from a large cohort with subgroup analyses. Int J Cancer. 2015;137(4):819-25. doi:10.1002/ijc.29449.

18. Huang Y, Liao H, Zhang Y, Yuan R, Wang F, Gao Y, Wang P, Du Z. Prognostic value of tumor-infiltrating FoxP3+ T cells in gastrointestinal cancers: a meta analysis. PLoS One. 2014;9(5):e94376. doi:10.1371/journal.pone.0094376.

19. Hou J, Yu Z, Xiang R, Li C, Wang L, Chen S, Li Q, Chen M, Wang L. Correlation between infiltration of $\mathrm{FOXP}^{+}$regulatory $\mathrm{T}$ cells and expression of $\mathrm{B} 7-\mathrm{H} 1$ in the tumor tissues of gastric cancer. Exp Mol Pathol. 2014;96(3):284-91. doi:10.1016/j. yexmp.2014.03.005. 